\title{
Study on the Physical Basis of Wave-Particle Duality: Modelling the Vacuum as a Continuous Mechanical Medium
}

\author{
Donald C. Chang', Yi-Kuen Lee ${ }^{2}$ \\ ${ }^{1}$ Division of LIFS, Hong Kong University of Science and Technology, Hong Kong, China \\ ${ }^{2}$ Department of Mechanical \& Aerospace Engineering, Hong Kong University of Science and Technology, \\ Hong Kong, China \\ Email: bochang@ust.hk
}

Received 25 May 2015; accepted 13 July 2015; published 16 July 2015

Copyright (C) 2015 by authors and Scientific Research Publishing Inc.

This work is licensed under the Creative Commons Attribution International License (CC BY).

http://creativecommons.org/licenses/by/4.0/

(c) (i) Open Access

\begin{abstract}
One great surprise discovered in modern physics is that all elementary particles exhibit the property of wave-particle duality. We investigated this problem recently and found a simple way to explain this puzzle. We proposed that all particles, including massless particles such as photon and massive particles such as electron, can be treated as excitation waves in the vacuum, which behaves like a physical medium. Using such a model, the phenomenon of wave-particle duality can be explained naturally. The key question now is to find out what kind of physical properties this vacuum medium may have. In this paper, we investigate if the vacuum can be modeled as an elastic solid or a dielectric medium as envisioned in the Maxwell theory of electricity and magnetism. We show that a similar form of wave equation can be derived in three cases: (1) By modelling the vacuum medium as an elastic solid; (2) By constructing a simple Lagrangian density that is a 3-D extension of a stretched string or a vibrating membrane; (3) By assuming that the vacuum is a dielectric medium, from which the wave equation can be derived directly from Maxwell's equations. Similarity between results of these three systems suggests that the vacuum can be modelled as a mechanical continuum, and the excitation wave in the vacuum behaves like some of the excitation waves in a physical medium.
\end{abstract}

\section{Keywords}

Vacuum, Wave-Particle Duality, Matter Wave, Elementary Particle, Vacuum Medium 


\section{Introduction}

It is well known that all particles in nature have a peculiar property called the wave-particle duality [1] [2]. That is, a particle (such as a photon) can behave like a wave and also like a corpuscular object. More than three hundred years ago, there was a debate between Newton and Huygens about the behavior of light. Newton thought that light behaved like a particle [3], while Huygens thought that light is a wave [4]. Later in the $19^{\text {th }}$ century, Maxwell and Hertz demonstrated that light is indeed an electromagnetic wave [5]. This was a monumental discovery. However, Einstein in 1905 studied the photoelectric effect [6] and showed that light behaves more like a particle. So, we can only accept that light has both particle properties and wave properties. This is called "waveparticle duality”.

Interestingly, it was later discovered that not only light exhibits wave-particle duality, other particles, such as electrons, also show wave-particle duality [7] [8]. In fact, it was shown by Louis de Broglie that the momentum of an electron is inversely proportional of its wavelength [9]. One can even take advantage of the wave property of electrons to make an electron microscope. Later, it was discovered experimentally that particles other than electrons, such as neutrons, also exhibit wave-particle duality [10]. More surprisingly, it was found that even atoms [11] and molecules [12], which make up matters, also possess properties of wave in diffraction experiments. These results strongly suggested that matter is made up by waves.

The question then is: Is the wave making up matter a physical wave or a probability wave? In the conventional theory of quantum mechanics, particles are treated as pointed objects. The wave property is only related to probability. That is, the quantum wave function gives the probability of finding a particle at a particular space and time. This interpretation is called "the Copenhagen Interpretation” [13], which had been the standard teaching of quantum mechanics for a long time. Nevertheless, some well-known physicists still had reservations about this interpretation. For example, Einstein had expressed his disapproval in a famous comment: "God does not play dice!” [14]

We think it is more natural to regard the particle as a physical wave, more exactly, the excitation wave of the vacuum medium. This excitation looks like a wave in a microscopic view; but in a macroscopic view, it looks like a particle. This phenomenon is well known to physicists studying condensed matter. For example, the phonon is a vibrational excitation wave in a mechanical medium; but it can be treated as a quasi-particle in a macroscopic view. We know at least one particle, i.e., the photon, is indeed an excitation wave. In fact, we know it is an electromagnetic wave in the microscopic view. But it can also exhibit the property of a particle in a macroscopic view, such as in the case of the photo-electric effect.

We showed previously that this physical wave hypothesis can give remarkable results [15]-[17]. The basic assumptions of our model are:

(a) The particle is a physical wave rather than a probability wave; it behaves just like a quasi-particle.

(b) The vacuum is a continuous medium, which can be excited by an energetic stimulation. The excitation wave can travel within this medium in long distance without energy loss (just like electrons in a superconductor or phonons in a superfluid).

(c) Different excitation waves of the vacuum medium make up different types of particles observed in the physical world. Both fermions and bosons are excitation waves of the same vacuum medium.

Based on these assumptions, one can explain a large number of quantum observations [15]-[17]. The key question now is how to determine the physical properties of the vacuum medium. This question had been explored before. In the $19^{\text {th }}$ century, many scientists believed that the empty space between matters was filled with a medium called "aether". They assumed that aether is the medium that carries the electro-magnetic wave. This hypothesis, however, became disfavored later with the development of the special theory of relativity. The major reason for this disfavoring is because the aether model failed to explain the results of the Michelson-Morely experiment, which did not detect any relative movement between aether and the planet Earth.

Here, we would like to point out the difference between our model and the aether hypothesis. The aether was hypothesized to fill in the space between matters: it is a medium for the propagation of light only [18]. The aether had nothing to do with matter itself. In our model, not only electromagnetic waves (photons), but all particles, including sub-atomic particles that make up matters in the universe, are excitation waves of the vacuum medium. Thus, when matters (such as a planet) move through the vacuum medium, there is no resistance. There is no wonder that any attempt to detect the Earth's movement through the vacuum medium will fail. This explains the null results of experiments like those conducted by Michelson and Morely [19]. 
In order to make our model convincing, we need to address two basic questions: (1) What are the properties of the vacuum medium? (2) Can we derive the wave equation for the particle based on the physical properties of the vacuum? The purpose of this paper is trying to answer these questions. Here, we will consider three cases: First, we will show that, the vacuum can be modelled conceptually as an elastic solid, based on which the wave equation for its excitation waves can be derived. Second, we will treat the vacuum as a 3-dimensional mechanical medium which is an extension from a 1-D (stretched string) and 2-D (rubber membrane) systems. We show that we can derive the proper wave equation by constructing a Lagrangian density generalized from the Lagrangian densities of the 1-D and 2-D systems. Third, we will model the vacuum as a dielectric system as envisioned by Maxwell. The wave equation obtained in this case has very similar form as that of an elastic solid. Results of our investigation suggest that the vacuum indeed behave like a continuous mechanical medium. Implications of these findings will be discussed in details.

\section{Modelling the Vacuum as an Elastic Solid}

Here we will model the vacuum conceptually as an elastic solid. It is well known that many different types of excitation waves can be excited in such a medium. Some of these excitation waves behave similarly to that of a photon. In the following, we will show how such photon-like wave equations can be derived if one regards the vacuum medium as an elastic solid.

For an elastic solid body, the displacement and velocity fields of the differential solid element, $\mathrm{d} V(=\mathrm{d} x \mathrm{~d} y \mathrm{~d} z)$, are denoted as $r_{\mathrm{i}}$ and $u_{i}(i=1,2,3)$ (see Figure 1). The time derivative of the momentum (density $\rho \times$ velocity vector $u_{i}$ ) is equal to the summation of the surface/traction force $T_{i}$ and the body force $f_{i}$ applied. The traction force $T_{i}$ is known to be related to the second-order stress tensor $\sigma_{i j}[20]$ :

$$
T_{i}=\sigma_{i j} n_{j}
$$

where $\boldsymbol{n}=\left\{n_{i}\right\}$ is the normal unit vector with respect to the solid element. As shown in Appendix A, the movement of this solid element can be described by the following equation:

$$
\rho \frac{\partial u_{i}}{\partial t}+\rho u_{k} \frac{\partial u_{i}}{\partial x_{k}}=\frac{\partial \sigma_{i j}}{\partial x_{j}}+\rho f_{i}
$$

Here we use the convention of summing over repeated indices when they occur once as subscript and once as superscript. If the deformation of the elastic body is small, we can neglect the second-order terms. Recall that the velocity vector $u_{i}$ is the time derivative of the displacement $r_{i}$, we have

$$
\frac{\partial \sigma_{i j}}{\partial x_{j}}+\rho f_{i}=\rho \frac{\partial u_{i}}{\partial t}=\rho \frac{\partial^{2} r_{i}}{\partial t^{2}}
$$

If the material in the solid is linear, isotropic and the deformation is small, the strain tensor $e_{i j}$ can be related to the average of the deformation gradient $r_{i, j}$ [21],

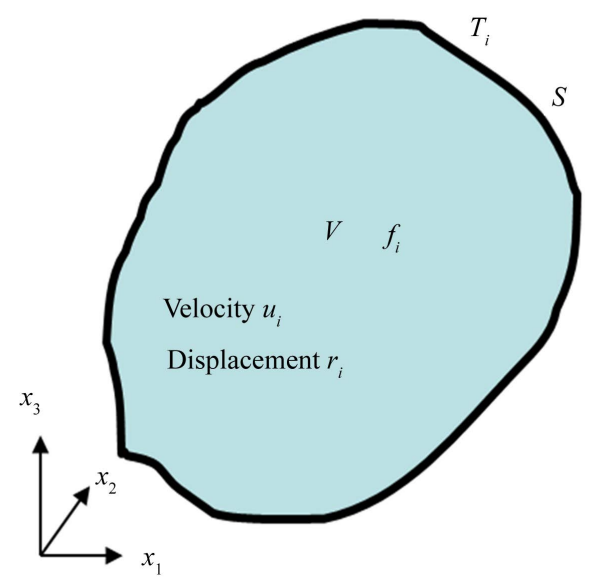

Figure 1. An elastic solid body with a volume of $V$ and a surface of $S$. 


$$
e_{i j}=\frac{1}{2}\left(r_{i, j}+r_{j, i}\right)
$$

where $r_{i, j}=\frac{\partial r_{i}}{\partial x_{j}}$. In addition, the stress tensor for isotropic material is known to be related to the strain tensor according to the generalized Hook's law with only two constants [20] [21]:

$$
\sigma_{i j}=\lambda e_{k k} \delta_{i j}+2 \mu e_{i j}=\lambda r_{k, k} \delta_{i j}+\mu\left(r_{i, j}+r_{j, i}\right)
$$

where $\lambda$ and $\mu$ are Lamé's first parameter and Lamé's second parameter. $\mu$ is also called the "shear modulus" [22]. Substituting Equation (5) into Equation (3), we can obtain the following equation (called the "Navier equation"), which is the fundamental governing equations for wave propagation in solid mechanics [21]:

$$
(\lambda+\mu) r_{j, j i}+\mu r_{i, j j}+\rho f_{i}=\rho \ddot{r}_{i}
$$

Since there is no external force applied to the vacuum, the body force $f_{i}$ is zero here. Using the vector notation of the gradient and divergence operators, we can re-write the tensor equation of Equation (6) as the following:

$$
(\lambda+\mu) \nabla(\nabla \cdot \boldsymbol{r})+\mu \nabla^{2} \boldsymbol{r}=\rho \frac{\partial^{2} \boldsymbol{r}}{\partial t^{2}}
$$

Based on Helmholtz decomposition theorem [23], a vector $\boldsymbol{r}$ can be decomposed into a curl-free component $\phi$ and a divergence-free component $\psi$.

$$
\boldsymbol{r}=\nabla \varphi+\nabla \times \psi,
$$

where $\nabla \cdot \boldsymbol{\psi}=0$. Substituting Equation (8) into Equation (7), we have

$$
(\lambda+\mu) \nabla(\nabla \cdot[\nabla \varphi+\nabla \times \boldsymbol{\psi}])+\mu \nabla^{2}[\nabla \varphi+\nabla \times \boldsymbol{\psi}]=\rho \frac{\partial^{2}}{\partial t^{2}}[\nabla \varphi+\nabla \times \psi]
$$

This equation can be rearranged to become

$$
\nabla\left\{(\lambda+2 \mu) \nabla^{2} \varphi-\rho \frac{\partial^{2} \varphi}{\partial t^{2}}\right\}+\nabla \times\left\{\mu \nabla^{2} \psi-\rho \frac{\partial^{2} \boldsymbol{\psi}}{\partial t^{2}}\right\}=0
$$

Equation (9) will be satisfied if each bracketed term is equal to zero. Therefore, we can get two uncoupled wave equations:

$$
\left\{\begin{array}{l}
(\lambda+2 \mu) \nabla^{2} \varphi-\rho \frac{\partial^{2} \varphi}{\partial t^{2}}=0 \\
\mu \nabla^{2} \psi-\rho \frac{\partial^{2} \psi}{\partial t^{2}}=0
\end{array}\right.
$$

Re-arranging the coefficients of Equations (10) and (11), we have

$$
\left\{\begin{array}{l}
\nabla^{2} \varphi-\frac{1}{c_{p}^{2}} \frac{\partial^{2} \varphi}{\partial t^{2}}=0 \\
\nabla^{2} \psi-\frac{1}{c_{s}^{2}} \frac{\partial^{2} \psi}{\partial t^{2}}=0
\end{array}\right.
$$

where $c_{p}=\sqrt{(\lambda+2 \mu) / \rho}$ is the velocity of the dilational wave (also called "primary wave/P wave") and $c_{s}=\sqrt{\mu / \rho}$ is the velocity of the transverse wave (also called "distortional wave/secondary wave/S wave/shear wave").

These wave equations have the identical form as that of the wave equation of light. We know that light is a transverse wave, so is the shear wave $\psi$ described in Equation (13). These similarities suggest that it is a good analogy for modelling the motion of the excitation wave in the vacuum as a transverse wave propagating in an elastic solid. Furthermore, Equation (13) clearly suggests that the value of the wave speed is associated with the 
physical properties of the medium.

\section{Modelling the Vacuum as a 3-Dimensional Continuous Mechanical System}

The wave equation as shown in Equation (13) or Equation (12) may be called the "four-dimensional Laplace equation". We can derive the same wave equation if we assume that the vacuum is a 3-dimensional isotropic medium. The key is to construct a proper Lagrangian density for the system. Then, we can apply the EulerLagrange equation based on Hamilton's principle. In order to determine what would be a proper Lagrangian density for the vacuum, we will examine what will happen in a 1-D system first. Then, we will extend the same analysis to a 2-D system. Finally, following the same extension method, we will obtain the generalized Lagrangian density for the 3-D continuum.

\subsection{Wave Motion in a 1-D System}

A good example of the 1-D continuum system can be a stretched string. Thus, in order to analyze the wave motion in a 1-D system, let us examine the propagation of wave along a string. For the string showed in the Figure 2 , the distance on the string is marked by the parameter $x$. The string has a total length of $l$. From classical mechanics, it is well known that the Lagrangian $(L)$ equals the kinetic energy $(T)$ minus the potential energy $(V)$. For a 1-D continuum, $L$ is an integration of the Lagrangian density $(\mathcal{L})$ along the string,

$$
L=T-V=\int_{0}^{l} \mathcal{L} \mathrm{d} x
$$

Let us denote the mass density of the string as $\rho$. The kinetic energy $(\Delta T)$ for a very small segment of the string (length of $\Delta x$ ) is

$$
\Delta T=\frac{1}{2}(\rho \Delta x)\left(\frac{\partial \phi}{\partial t}\right)^{2}
$$

For the entire string, the kinetic energy is

$$
T=\int_{0}^{l} \frac{1}{2} \rho\left(\frac{\partial \phi}{\partial t}\right)^{2} \mathrm{~d} x
$$

What about the potential energy $V$ ? Again, we will look at a small segment $\Delta x$ of the string, and determine the potential energy in this segment $(\Delta V)$. The string is stretched under tension $F_{1}$ between two points. For any parts of the string, the deviation from the equilibrium position is very small. As shown in Figure 2, the length of thestring over $\Delta x$ is stretched during the wave motion. This stretching length is denoted as $\Delta s$. Thus, the potentialenergy for this segment of the string is

$$
\Delta V=F_{1}(\Delta s-\Delta x)
$$

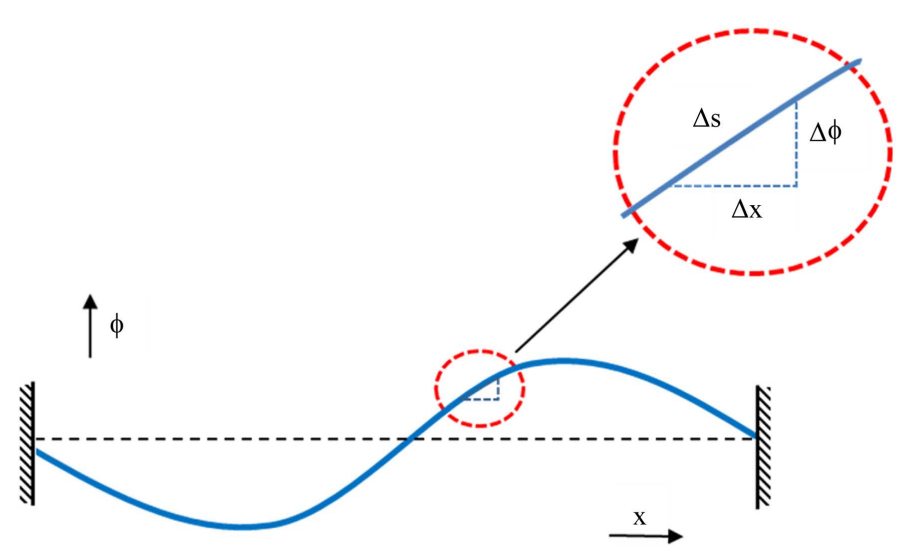

Figure 2. Wave propagation in a 1-D continuum system (a stretched string). 
From the inset in Figure 2, we can see

$$
\Delta s=\sqrt{\Delta x^{2}+\Delta \phi^{2}}=\Delta x\left[1+\frac{\Delta \phi^{2}}{\Delta x^{2}}\right]^{\frac{1}{2}}=\Delta x\left[1+\frac{1}{2}\left(\frac{\Delta \phi}{\Delta x}\right)^{2}+\cdots\right]
$$

When $\Delta \phi$ is small, we can ignore the higher terms. So,

$$
\Delta V=\frac{F_{1}}{2}\left(\frac{\partial \phi}{\partial x}\right)^{2} \Delta x
$$

and

$$
V=\int_{0}^{l} \frac{F_{1}}{2}\left(\frac{\partial \phi}{\partial x}\right)^{2} \mathrm{~d} x
$$

Substituting Equations (15) and (16) into Equation (14), we have

$$
L=\int_{0}^{l}\left[\frac{1}{2} \rho\left(\frac{\partial \phi}{\partial t}\right)^{2}-\frac{F_{1}}{2}\left(\frac{\partial \phi}{\partial x}\right)^{2}\right] \mathrm{d} x
$$

This implies

$$
\mathcal{L}=\frac{1}{2} \rho\left(\frac{\partial \phi}{\partial t}\right)^{2}-\frac{1}{2} F_{1}\left(\frac{\partial \phi}{\partial x}\right)^{2}
$$

Applying the Euler-Lagrange equation for 1-D system,

$$
\frac{\partial}{\partial t}\left(\frac{\partial \mathcal{L}}{\partial \dot{\phi}}\right)+\frac{\partial}{\partial x}\left(\frac{\partial \mathcal{L}}{\partial \phi^{\prime}}\right)=0
$$

where $\frac{\partial \phi}{\partial t}=\dot{\phi}, \frac{\partial \phi}{\partial x}=\phi^{\prime}$. We can obtain the wave equation,

$$
F_{1} \frac{\partial^{2} \phi}{\partial x^{2}}-\rho \frac{\partial^{2} \phi}{\partial t^{2}}=0
$$

or

$$
\frac{\partial^{2} \phi}{\partial x^{2}}-\frac{1}{c_{1}^{2}} \frac{\partial^{2} \phi}{\partial t^{2}}=0
$$

where $c_{1}=\sqrt{F_{1} / \rho}$. Equation (19A) is the wave equation for a 1-D system.

\subsection{Wave Equation in a 2-D System}

Now one can extend the 1-D system into a 2-D system, an example of which can be a stretched rubber membrane. We can follow the same procedure as that of the 1-dimensional string, except that the element to be considered is an area segment defined by $\Delta x \Delta y$. The Lagrangian for the 2-D system is

$$
L=T-V=\iint \mathcal{L} \mathrm{d} x \mathrm{~d} y
$$

Similar to the 1-D system, it can be shown that the kinetic energy $(\Delta T)$ and the potential energy $(\Delta V)$ over the area segment $\Delta x \Delta y$ are

and

$$
\Delta T=\frac{1}{2}(\rho \Delta x \Delta y)\left(\frac{\partial \phi}{\partial t}\right)^{2}
$$

$$
\Delta V=\frac{F_{2}}{2}\left[\left(\frac{\partial \phi}{\partial x}\right)^{2}+\left(\frac{\partial \phi}{\partial y}\right)^{2}\right] \Delta x \Delta y
$$


where $\rho$ is the density of the membrane per unit area. Substituting the above equations into Equation (20), we have

$$
L=\iint \mathcal{L} \mathrm{d} x \mathrm{~d} y=T-V=\iint\left\{\frac{1}{2} \rho\left(\frac{\partial \phi}{\partial t}\right)^{2}-\frac{F_{2}}{2}\left[\left(\frac{\partial \phi}{\partial x}\right)^{2}+\left(\frac{\partial \phi}{\partial y}\right)^{2}\right]\right\} \mathrm{d} x \mathrm{~d} y
$$

Then,

$$
\mathcal{L}=\frac{1}{2} \rho\left(\frac{\partial \phi}{\partial t}\right)^{2}-\frac{1}{2} F_{2}\left[\left(\frac{\partial \phi}{\partial x}\right)^{2}+\left(\frac{\partial \phi}{\partial y}\right)^{2}\right]
$$

Applying the generalized Euler-Lagrange equation [24], we can obtain

$$
F_{2}\left(\frac{\partial^{2} \phi}{\partial x^{2}}+\frac{\partial^{2} \phi}{\partial y^{2}}\right)-\rho \frac{\partial^{2} \phi}{\partial t^{2}}=0
$$

or

$$
\left(\frac{\partial^{2} \phi}{\partial x^{2}}+\frac{\partial^{2} \phi}{\partial y^{2}}\right)-\frac{1}{c_{2}^{2}} \frac{\partial^{2} \phi}{\partial t^{2}}=0
$$

where $c_{2}=\sqrt{F_{2} / \rho}$. This is the wave equation of the 2-D continuum system.

\subsection{Wave Equation in a 3-D System}

Now, by applying same procedures as the last two sub-sections, we can extend the 2-D system into the 3-D system. For a 3-D system, the Lagrangian density is defined as

$$
L=T-V=\iiint \mathcal{L} \mathrm{d} x \mathrm{~d} y \mathrm{~d} z
$$

By generalization of the 1-D and 2-D systems, it can be shown that

$$
L=\iiint\left\{\frac{1}{2} \rho\left(\frac{\partial \phi}{\partial t}\right)^{2}-\frac{F_{3}}{2}\left[\left(\frac{\partial \phi}{\partial x}\right)^{2}+\left(\frac{\partial \phi}{\partial y}\right)^{2}+\left(\frac{\partial \phi}{\partial z}\right)^{2}\right]\right\} \mathrm{d} x \mathrm{~d} y \mathrm{~d} z
$$

Thus,

$$
\mathcal{L}=\frac{1}{2} \rho\left(\frac{\partial \phi}{\partial t}\right)^{2}-\frac{1}{2} F_{3}\left[\left(\frac{\partial \phi}{\partial x}\right)^{2}+\left(\frac{\partial \phi}{\partial y}\right)^{2}+\left(\frac{\partial \phi}{\partial z}\right)^{2}\right]
$$

Once we know the Lagrangian density of the 3-D system, we can apply the generalized Euler-Lagrange equation to obtain the equation of motion,

$$
F_{3}\left(\frac{\partial^{2} \phi}{\partial x^{2}}+\frac{\partial^{2} \phi}{\partial y^{2}}+\frac{\partial^{2} \phi}{\partial z^{2}}\right)-\rho \frac{\partial^{2} \phi}{\partial t^{2}}=0
$$

or,

$$
\nabla^{2} \phi-\frac{1}{c_{3}^{2}} \frac{\partial^{2} \phi}{\partial t^{2}}=0
$$

where the coefficient $c_{3}=\sqrt{F_{3} / \rho}$ is the speed of the wave. This is the general equation describing the motion of excitation waves in the 3-dimentional medium. If the vacuum can be modelled as such a medium and a free particle is an excitation wave of the vacuum, then Equation (25) will become the wave equation for the free particle.

\section{Modelling the Vacuum as a Dielectric Medium}

In Sections 2 and 3, we derived the wave equation by modelling the vacuum as an elastic solid or a 3-D mechanical 
continuum. They yield wave equations very similar to the wave equation of light. These results suggest that the vacuum medium could be treated as an elastic solid, and the propagating wave is just an excitation of the medium. However, there is a problem in regarding the vacuum as a true solid, since there is no rest mass in the vacuum. Thus, we cannot apply Newton's law to calculate the kinetic energy of the vacuum. This means that, the Lagrangian density of the vacuum cannot be the same as that of the elastic solid. Therefore, the discussions in Section 2 and Section 3 can only serve as an analogy to demonstrate how a wave can be generated in a continuous mechanical medium. To study the wave propagation in the vacuum system, one needs to use a more realistic model.

We think the vacuum is more like a dielectric medium, which is responsible for giving rise to the electromagnetic field. The physical nature of such a medium can be investigated by examining the assumptions behind the derivation of Maxwell's equations. In the $19^{\text {th }}$ century, there had been extensive studies on the physical natures of the electro-magnetic fields. It was strongly believed that the propagation of the electro-magnetic waves require a dielectric medium. We should take note from these classical studies.

In 1861-1862, Maxwell published his famous equations on electric and magnetic fields based on the earlier works of Coulomb, Ampère, Faraday and others. The early versions of Maxwell's equations [5] were as the following:

$$
\left\{\begin{array}{l}
\nabla \times \boldsymbol{E}=-\frac{\partial \boldsymbol{B}}{\partial t} \quad \text { Faraday's Law } \\
\nabla \times \boldsymbol{H}=\boldsymbol{J} \quad \text { Ampère's Law } \\
\nabla \cdot \varepsilon \boldsymbol{E}=\rho_{e} \quad \text { Coulomb's Law (or Gausss Law) } \\
\nabla \cdot \boldsymbol{B}=0 \quad \text { Gauss' Law for Magnetism }
\end{array}\right.
$$

Here, $\boldsymbol{E}$ is electric field; $\boldsymbol{J}$ is electric current density; $\boldsymbol{B}$ is magnetic flux density, $\boldsymbol{H}$ is magnetic field, and $\varepsilon$ is the permittivity. It is known that

$$
\boldsymbol{B}=\mu \boldsymbol{H}
$$

where $\mu$ is the permeability. In free space, the permittivity is $\varepsilon_{0}$ and the permeability is $\mu_{0}$.

This original set of Maxwell equations has a problem. In order to satisfy the condition of conservation of charge, the system should obey the continuity equation

$$
\nabla \cdot \boldsymbol{J}=-\frac{\partial \rho_{e}}{\partial t}
$$

But from Equation (27),

$$
\nabla \cdot(\nabla \times \boldsymbol{H})=\nabla \cdot \boldsymbol{J}
$$

which implies

$$
\nabla \cdot \boldsymbol{J}=0
$$

This means that Equation (27) did not satisfy the continuity equation. Thus, Maxwell was not satisfied with it. To correct this problem, Maxwell thought an additional term $\partial \boldsymbol{D} / \partial t$ must be added into the right hand-side of Equation (27) [5]. The justification for adding this new term was that, in a dielectric material, there are both positive and negative charges embedded within the medium. So, when the dielectric medium is exposed to an electric field, it will induce a displacement of the dielectric charges (this is called $\boldsymbol{D}$ ). A time variation of the displaced charge should produce a displacement current $\left(\boldsymbol{J}_{\mathrm{d}}\right)$, which can also affect the magnetic field. So that in Equation (27), one should consider not only the externally applied current $\boldsymbol{J}$, but also the internally induced displacement current $\boldsymbol{J}_{\mathrm{d}}$. That is

$$
\boldsymbol{J}_{\text {total }}=\boldsymbol{J}+\boldsymbol{J}_{\mathrm{d}}=\boldsymbol{J}+\frac{\partial \boldsymbol{D}}{\partial t}
$$

Then, Equation (27) now becomes

$$
\nabla \times \boldsymbol{H}=\boldsymbol{J}+\frac{\partial \boldsymbol{D}}{\partial t}
$$


Taking the divergence of both sides of Equation (27A),

$$
\nabla \cdot(\nabla \times \boldsymbol{H})=\nabla \cdot \boldsymbol{J}+\frac{\partial(\nabla \cdot \boldsymbol{D})}{\partial t}
$$

It is known that $\boldsymbol{D}=\varepsilon \boldsymbol{E}$.Using Equation (28), the above equation becomes

$$
\nabla \cdot \boldsymbol{J}+\frac{\partial \rho_{e}}{\partial t}=0
$$

This agrees exactly with the continuity equation.

With the addition of the displacement current, the final form of the Maxwell's equations becomes:

$$
\left\{\begin{array}{l}
\nabla \times \boldsymbol{E}=-\frac{\partial \boldsymbol{B}}{\partial t} \\
\nabla \times \boldsymbol{B}=\mu \varepsilon \frac{\partial \boldsymbol{E}}{\partial t}+\boldsymbol{J} \\
\nabla \cdot \varepsilon \boldsymbol{E}=\rho_{e} \\
\nabla \cdot \boldsymbol{B}=0
\end{array}\right.
$$

From Equation (29), it is implied

$$
\boldsymbol{B}=\nabla \times \boldsymbol{A}
$$

One may notice that the vector potential $\boldsymbol{A}$ in Equation (32) is not unique. One can replace $\boldsymbol{A}$ with $\boldsymbol{A}+\nabla \boldsymbol{X}$, (where $\mathcal{X}$ is any scalar function) and $\boldsymbol{B}$ will remain the same. This freedom is an example of "gauge transformation".

At this point, we can write down Maxwell's equations in the vacuum. In a free space, $\boldsymbol{J}=0$, and $\rho_{e}=0$; also $\mu=\mu_{0}, \varepsilon=\varepsilon_{0}$. Thus, Maxwell's equations in the free space now become:

$$
\left\{\begin{array}{l}
\nabla \times \boldsymbol{E}=-\frac{\partial \boldsymbol{B}}{\partial t} \\
\nabla \times \boldsymbol{B}=\mu_{0} \varepsilon_{0} \frac{\partial \boldsymbol{E}}{\partial t} \\
\nabla \cdot \varepsilon_{0} \boldsymbol{E}=0 \\
\nabla \cdot \boldsymbol{B}=0
\end{array}\right.
$$

From the above Maxwell's equations, we can derive the wave equation of the electro-magnetic wave in a free space. It turns out that this wave equation is the same as that describing the propagation of light. This remarkable result allowed Maxwell to identify light as a kind of electro-magnetic wave. This conclusion was later confirmed in experiments conducted by Hertz.

We must point out that, the above Maxwell's equations was based on the implicit assumption that the vacuum behaves like a dielectric medium. By comparing Equation (27C) with Equation (27A), it is clear that the right hand-side of Equation (27C) is originated from the displacement current in Equation (27A). That means although the current $\boldsymbol{J}$ has vanished in free space, the displacement current still remains. This is justifiable only if Maxwell's assumption that the vacuum behaves like a dielectric medium is correct. If the vacuum does not behave like a dielectric medium, one cannot explain the presence of the displacement current term in the right handside of Equation (27C)!

Now let us consider that: What is the Lagrangian density in the electro-magnetic system as described by Maxwell's equations? Previously, $\mathcal{L}$ is defined as $T-V$ in a mechanical system. This definition does not apply directly for the vacuum medium, since the vacuum has no rest mass and thus it is difficult to define its kinetic energy ( $T$ ). However, one can still construct a proper Lagrangian density that will give rise to the correct form of Maxwell's equations. In fact, the Lagrangian density for the electro-magnetic field in the free space [25] is known to be

$$
\mathcal{L}=\frac{1}{2}\left(\boldsymbol{E}^{2}-\boldsymbol{B}^{2}\right)
$$




$$
=-\frac{1}{4} \sum_{\mu} \sum_{v}\left(\partial_{\mu} A_{v}-\partial_{\nu} A_{\mu}\right)\left(\partial^{\mu} A^{\nu}-\partial^{\nu} A^{\mu}\right)
$$

Here we use the covariant-contravariant four vector convention, the index $\mu$ and $v=0,1,2,3$;

$\partial_{\mu}=\left(\frac{1}{c} \frac{\partial}{\partial t}, \frac{\partial}{\partial x}, \frac{\partial}{\partial y}, \frac{\partial}{\partial z}\right), \partial^{\mu}=\left(\frac{1}{c} \frac{\partial}{\partial t},-\frac{\partial}{\partial x},-\frac{\partial}{\partial y},-\frac{\partial}{\partial z}\right), A^{v}=\left(\phi, A_{x}, A_{y}, A_{z}\right), A_{v}=\left(\phi,-A_{x},-A_{y},-A_{z}\right)$, where $\phi$ is the scalar potential and $\boldsymbol{A}$ is the vector potential. One can show that this Lagrangian density can give rise to the complete set of Maxwell's equations using Hamilton's principle [25].

From Maxwell's equations in the free space, one can show that both the electric field and the magnetic field can propagate as an oscillating transverse wave in the vacuum [5]. Also, using Maxwell's equations in the free space, one can derive a general wave equation in terms of the vector potential $\boldsymbol{A}$ :

$$
\nabla^{2} \boldsymbol{A}-\frac{1}{c^{2}} \frac{\partial^{2} \boldsymbol{A}}{\partial t^{2}}=0
$$

where $c=1 / \sqrt{\mu_{0} \varepsilon_{0}}$ is equal to the speed of light. At this point, one can easily see that there are certain common features between the mechanical systems discussed in Section 3 and the electro-magnetic field system discussed here. First, we know that the Lagrangian density for a mechanical system is consisted of quadratic terms of the first derivative of the field parameter $\left(\partial_{\mu} \phi\right)$. Here in Equation (33), we see that the Lagrangian density for the electro-magnetic system is also consisted of "quadratic terms" of the first derivative of the vector potential $\left(\partial_{\mu} A_{v}\right)$. Second, if we use $\psi$ to represent the spatial components of the vector potential $A$, i.e., $A_{\mu} \rightarrow \psi$. Equation (34) would become

$$
\nabla^{2} \psi-\frac{1}{c^{2}} \frac{\partial^{2} \psi}{\partial t^{2}}=0
$$

This looks exactly like the wave equations (Equation (25)) derived for a 3-D continuum in Section 3. (In fact, $\psi$ can be any linear combination of $A_{\mu}$, and Equation (35) will still hold.) So, there is strong similarity between wave propagation in the vacuum and wave propagation in a continuous mechanical system.

\section{Discussions}

In this work, we conducted a comparative study of the motion of excitation waves in three different systems: (a) the vacuum is modelled as an elastic solid (discussed in Section 2); (b) the vacuum is modelled as a general 3-D continuum (discussed in Section 3); and (c) the vacuum is modelled as a dielectric medium as envisioned by Maxwell. The results are remarkably alike. The forms of their wave equations are identical (see Equations (13), (25), (35)). This similarity strongly suggests that the vacuum behaves like a continuous mechanical system, and the excitation wave in the vacuum follows similar rules as the excitation waves in a physical medium.

It is known in condense matter physics that the excitation wave in a solid can behave like a particle (called "phonon"). Such particle-like excitations are classified as "quasi-particles". Light, as an excitation wave in the vacuum, can be regarded as a real particle (called "photon"). The particle properties of photon were clearly demonstrated in the studies of photo-electric effect and Compton scattering. In our previous papers, we showed it is well justified to hypothesize that all particles in nature are different excitation modes of the vacuum medium. They all obey the same wave equation; different solutions can represent different particles. One can identify the key particle properties, including energy, momentum, and rest mass $(m)$, from the parameters of their wave functions. (For details, see Ref. [15]). Furthermore, one can naturally derive the Klein-Gordon equation (for a scalar particle of specific $m$ ) from the wave equation of the vacuum. We show that it is a simplified wave equation which describes only the variation of the excitation wave in the longitudinal direction (i.e., parallel to the particle's trajectory) [15]. Once the Klein-Gordon equation is derived, one can then easily obtain the Dirac equation to describe the motion of a fermion, such as electron [26]. Then, the Schrödinger equation can be derived from the Dirac equation under the condition when the Coulomb potential energy and kinetic energy are small in comparison to the rest mass of the particle [27].

Results of this work suggest that a free particle travelling through the vacuum is similar to an excitation wave propagating through a physical medium. Our work suggests that the physical meaning of the wave function is not just related to the probability of finding a particle; it can represent a real physical quantity. In fact, from 
Equations (13), (25), (35), one can see that the wave functions in all three model systems are closely related with the perturbation of the "field potential", which is connected with the "strain" of the medium, (i.e., a very tiny displacement of the medium elements from its equilibrium position) (See Equation (8)). As we have shown in the elastic solid, this strain is related to a stress tensor of the medium. Similarly, in the study of classical electrodynamics, it is well known that the electro-magnetic field also possesses a stress tensor (called the "Maxwell stress tensor") [28]. So, there is a close analogy between the wave motion in the vacuum and the wave motion in the elastic solid.

We would like to add that our hypothesis regarding the vacuum as a physical medium is not without basis. Investigation on the properties of the vacuum has a very long history [18]. Although the word "vacuum" implies that the space is filled with emptiness, many well-known physicists (including: Isaac Newton, Robert Boyle, Christiaan Huygens, Augustin-Louis Cauchy, James Maxwell, George Stokes, and Hendrik Lorentz) did not believe this is true [18]. Before the end of the $19^{\text {th }}$ century, most scientists thought the space between matters is filled with a magical dielectric medium called "aether". Many experiments were designed to verify the existence of this hypothetical aether. These experiments, however, all failed. This failure caused scientists in the early $20^{\text {th }}$ century to reject the aether hypothsis. So for a while, the vacuum was regarded as pure empty space. But later as quantum field theory was developed, people discovered that the vacuum can no longer be treated as emptiness. Instead, the vacuum was found to be very complicated. For example, according to the Dirac theory, there were an infinite number of negative-energy electrons at any point of the space [29] [30]. Before excitation, all energy levels for such negative-energy electrons are completely filled up. When the system is excited, some of the negative-energy electron will gain energy and become positive-energy electron. The hole left in the sea of negativeenergy electron then behaves like an anti-particle of the electron (i.e., positron).

Later, it was discovered in experiments using particle accelerators that many new particles can be created from collision. In order to explain these observations, it was assumed that the vacuum is full of all sorts of virtual particle \& anti-particle pairs [31]. Upon excitation, these virtual particles will become real particles. So again, the vacuum is very complicated.

The above problems can be avoided if we assume that particles are excitations of the vacuum medium and the wave function represents a real physical wave. In this way, it is easy to see that excitation waves can be created or annihilated in any location of the medium. There is no need to assume an infinite sea of negative-energy electrons. We can completely remove the assumption of the existence of unlimited amount of virtual particle pairs in the vacuum.

\section{Acknowledgements}

We would like to thank Ms. Lan Fu for her valuable assistance in preparing this manuscript. In this collaboration, the analysis of wave motion in an elastic solid was mainly contributed by YKL, while DCC was mainly responsible for the overall hypothesis of this work.

\section{References}

[1] Messiah, A. (1965) Quantum Mechanics. John Wiley \& Sons, New York, 45-59.

[2] Selleri, F. (1992) Wave-Particle Duality. Plenum Press, New York. http://dx.doi.org/10.1007/978-1-4615-3332-0

[3] Newton, I. (1704) Opticks: Or, a Treatise of the Reflexions, Refractions, Inflexions and Colours of Light. London.

[4] Huygens, C. (1690) Traité De La Lumière. Pieter van der Aa, Leiden.

[5] Longair, M.S. (1984) Theoretical Concepts in Physics: An Alternative View of Theoretical Reasoning in Physics for Final-Year Undergraduates. Cambridge University Press, Cambridge, New York, 37-59.

[6] Einstein, A. (1905) Annalen der Physik, 17, 132-147. http://dx.doi.org/10.1002/andp.200590004

[7] Davisson, C.J. and Germer, L.H. (1927) Nature, 119, 558-560. http://dx.doi.org/10.1038/119558a0

[8] Thomson, G.P. and Reid, A. (1927) Nature, 119, 890. http://dx.doi.org/10.1038/119890a0

[9] De Broglie, L. (1924) Recherchessur la théorie des quanta. Ph.D. Thesis, L’Université de Paris, Paris.

[10] Halban, H.V.J. and Preiswerk, P. (1936) Preuveexpérimentale de la diffraction des neutrons. Comptes Rendus de l'Académie des Sciences, Paris, 203, 73-75.

[11] Estermann, I. and Stern, O. (1930) Zeitschrift Für Physik, 61, 95-125. http://dx.doi.org/10.1007/BF01340293 
[12] Arndt, M., Nairz, O., Vos-Andreae, J., Keller, C., Van der Zouw, G. and Zeilinger, A. (1999) Nature, 401, $680-682$. http://dx.doi.org/10.1038/44348

[13] Messiah, A. (1965) Quantum Mechanics. Volume 1, John Wiley \& Sons, New York, 47-48.

[14] Einstein, A. and Born, M. (1971) The Born-Einstein Letters. Walker and Company, New York.

[15] Chang, D.C. (2013) Journal of Modern Physics, 4, 21-30. http://dx.doi.org/10.4236/jmp.2013.411A1004

[16] Chang, D.C. (2005) http://arxiv.org/abs/physics/0505010.

[17] Chang, D.C. (2004) http://arxiv.org/abs/physics/0404044.

[18] Whittaker, E. (1951) A History of the Theories of Aether and Electricity. Thomas Nelson and Sons Ltd., London.

[19] Michelson, A.A. and Morley, E.W. (1887) American Journal of Science, 34, 333-345. http://dx.doi.org/10.2475/ajs.s3-34.203.333

[20] Reismann, H. and Pawlik, P.S. (1980) Elasticity: Theory and Applications. John Wiley and Sons, New York.

[21] Fung, Y.C. (1977) A First Course in Continuum Mechanics. Prentice-Hall, Upper Saddle River.

[22] Salencon, J. (2001) Handbook of Continuum Mechanics: General Concepts, Thermoelasticity. Springer Science \& Business Media, New York, 333. http://dx.doi.org/10.1007/978-3-642-56542-7

[23] Arfken, G.B. and Weber, H.J. (1995) Mathematical Methods for Physicists. Cambridge University Press, Cambridge.

[24] Weinstock, R. (1952) Calculus of Variations with Applications to Physics and Engineering. McGraw-Hill, New York.

[25] Cottingham, W.N. and Greenwood, D.A. (1998) An Introduction to the Standard Model of Particle Physics. Cambridge University Press, Cambridge, 37-47.

[26] Sakurai, J.J. (1973) Advanced Quantum Mechanics. Addison-Wesley, Reading, 78-89.

[27] Cottingham, W.N. and Greenwood, D.A. (1998) An Introduction to the Standard Model of Particle Physics. Cambridge University Press, Cambridge, 72.

[28] Stratton, J.A. (1941) Electromagnetic Theory. McGraw-Hill, New York.

[29] Dirac, P.A.M. (1981) The Principles of Quantum Mechanics. Clarendon Press, Oxford, 253-275.

[30] Hey, T. and Walters, P. (2003) The New Quantum Universe. Cambridge University Press, Cambridge, 229. http://dx.doi.org/10.1017/cbo9780511818752

[31] Dodd, J.E. (1984) The Idea for Particle Physics: An Introduction for Scientists. Cambridge University Press, Cambridge, 30-31.

[32] Graff, K.F. (1975) Wave Motion in Elastic Solids. Clarendon Press, Oxford. 


\section{Appendix A: Wave Motion in an Elastic Solid}

For an elastic solid body with a volume of $V$ and a surface of $S$ as shown in Figure 1, the displacement and velocity fields of the differential solid element, $\mathrm{d} V(=\mathrm{d} x \mathrm{~d} y \mathrm{~d} z)$, are denoted as $r_{\mathrm{i}}$ and $u_{i}(i=1,2,3)$. The time derivative of the momentum (density $\rho \times$ velocity vector $u_{i}$ ) is equal to the summation of all the surface/traction force $T_{i}$ and all the body force $f_{i}$ applied [20] [32]:

$$
\frac{D}{D t} \int_{V} \rho u_{i} \mathrm{~d} V=\int_{S} T_{i} \mathrm{~d} s+\int_{V} \rho f_{i} \mathrm{~d} V
$$

(where $\frac{D}{D t}$ is the "material derivative”). The traction force $T_{i}$ can be related to the second-order stress tensor $\sigma_{i j}[20]:$

$$
T_{i}=\sigma_{i j} n_{j}
$$

where $\boldsymbol{n}=\left\{n_{i}\right\}$ is the normal unit vector with respect to the surface $S$. Here we have adopted the convention of summing over repeated indices when they occur once as subscript and once as superscript. Substitute Equation (A2) into Equation (A1),

$$
\frac{D}{D t} \int_{V} \rho u_{i} \mathrm{~d} V=\int_{S} \sigma_{i j} n_{j} \mathrm{~d} s+\int_{V} \rho f_{i} \mathrm{~d} V
$$

Using the divergence theorem (Green’s theorem) [23], we can convert the surface integral for the first term on the right hand side into the volume integral. Assuming the volume of the system will not change in the wave propagation, we have

$$
\int_{V} \frac{\mathrm{d}}{\mathrm{d} t}\left(\rho u_{i}\right) \mathrm{d} V=\int_{V} \frac{\partial \sigma_{i j}}{\partial x_{j}} \mathrm{~d} V+\int_{V} \rho f_{i} \mathrm{~d} V
$$

Recall that $\frac{\mathrm{d}}{\mathrm{d} t}=\frac{\partial}{\partial t}+u_{k} \frac{\partial}{\partial x_{k}}$ and apply the condition of incompressibility, $\nabla \cdot u=0$, we have

$$
\int_{V}\left[\frac{\partial}{\partial t}\left(\rho u_{i}\right)+\frac{\partial}{\partial x_{k}}\left(\rho u_{i} u_{k}\right)\right] \mathrm{d} V=\int_{V}\left[\frac{\partial \sigma_{i j}}{\partial x_{j}}+\rho f_{i}\right] \mathrm{d} V
$$

Move all the terms to the left hand side

$$
\int_{V}\left[\frac{\partial}{\partial t}\left(\rho u_{i}\right)+\frac{\partial}{\partial x_{k}}\left(\rho u_{i} u_{k}\right)-\frac{\partial \sigma_{i j}}{\partial x_{j}}-\rho f\right] \mathrm{d} V=0
$$

The above equation can be satisfied if the bracketed term is zero,

$$
\frac{\partial}{\partial t}\left(\rho u_{i}\right)+\frac{\partial}{\partial x_{k}}\left(\rho u_{i} u_{k}\right)=\frac{\partial \sigma_{i j}}{\partial x_{j}}+\rho f_{i}
$$

Applying the differentiation to the two terms on the left hand side of Equation (A5), and doing a simple rearrangement:

$$
\rho \frac{\partial u_{i}}{\partial t}+u_{i}\left[\frac{\partial \rho}{\partial t}+\frac{\partial}{\partial x_{k}}\left(\rho u_{k}\right)\right]+\rho u_{k} \frac{\partial u_{i}}{\partial x_{k}}=\frac{\partial \sigma_{i j}}{\partial x_{j}}+\rho f_{i}
$$

The bracket in the left hand side of Equation (A6) should be zero because it is the equation of continuity: $\frac{\partial \rho}{\partial t}+\frac{\partial}{\partial x_{k}}\left(\rho u_{k}\right)=0$. Therefore, Equation (A6) can be simplified as follows:

$$
\rho \frac{\partial u_{i}}{\partial t}+\rho u_{k} \frac{\partial u_{i}}{\partial x_{k}}=\frac{\partial \sigma_{i j}}{\partial x_{j}}+\rho f_{i}
$$

This is Equation (2) in the main text. 